\title{
Fluid Discrimination Based on Frequency-Dependent AVO Inversion with the Elastic Parameter Sensitivity Analysis
}

\author{
Pu Wang $\mathbb{D}^{1},{ }^{1}$ Jingye Li $\mathbb{D}^{1}{ }^{1}$ Xiaohong Chen, ${ }^{1}$ Kedong Wang, and Benfeng Wang $\mathbb{D}^{2}$ \\ ${ }^{1}$ State Key Laboratory of Petroleum Resources and Prospecting, National Engineering Laboratory for Offshore Oil Exploration, \\ China University of Petroleum-Beijing, Beijing 102249, China \\ ${ }^{2}$ Tongji University, State Key Laboratory of Marine Geology, School of Ocean and Earth Science, Institute for Advanced Study, \\ Shanghai 200092, China
}

Correspondence should be addressed to Jingye Li; ljy3605@sina.com

Received 15 August 2019; Revised 15 October 2019; Accepted 21 November 2019; Published 18 December 2019

Academic Editor: Mohammad Sarmadivaleh

Copyright (C) $2019 \mathrm{Pu}$ Wang et al. This is an open access article distributed under the Creative Commons Attribution License, which permits unrestricted use, distribution, and reproduction in any medium, provided the original work is properly cited.

\begin{abstract}
Fluid discrimination is an extremely important part of seismic data interpretation. It plays an important role in the refined description of hydrocarbon-bearing reservoirs. The conventional AVO inversion based on Zoeppritz's equation shows potential in lithology prediction and fluid discrimination; however, the dispersion and attenuation induced by pore fluid are not fully considered. The relationship between dispersion terms in different frequency-dependent AVO equations has not yet been discussed. Following the arguments of Chapman, the influence of pore fluid on elastic parameters is analyzed in detail. We find that the dispersion and attenuation of Russell fluid factor, Lamé parameter, and bulk modulus are more pronounced than those of P-wave modulus. The Russell fluid factor is most prominent among them. Based on frequency-dependent AVO inversion, the uniform expression of different dispersion terms of these parameters is derived. Then, incorporating the P-wave difference with the dispersion terms, we obtain new $\mathrm{P}$-wave difference dispersion factors which can identify the gas-bearing reservoir location better compared with the dispersion terms. Field data application also shows that the dispersion term of Russell fluid factor is optimal in identifying fluid. However, the dispersion term of Russell fluid factor could be unsatisfactory, if the value of the weighting parameter associated with dry rock is improper. Then, this parameter is studied to propose a reasonable setting range. The results given by this paper are helpful for the fluid discrimination in hydrocarbon-bearing rocks.
\end{abstract}

\section{Introduction}

Rock physics is an effective tool for studying reservoir petrophysical properties from elastic and anelastic parameters [1]. Effective elastic media theory and poroelasticity theory are very important components of rock physics theories. The difference is that whether the mobility of pore fluid is considered or not [1]. The former considers the effects of pore geometry [2-4], instead of the effects of fluid flow on velocity. The Biot-Gassmann theory [5] is the research basis of most poroelasticity theories which are used to study the wave propagation in fluid-saturated rocks. The Gassmann theorem is proved to be valid for many types of rocks $[6,7]$, and it is used to predict P- and S-wave velocities in the lowfrequency band $[8,9]$. Due to the influence of pore fluid, dispersion and attenuation occur in the elastic parameters of rocks [10-12]. It is the same in the seismic frequency band, and some researchers have described the dispersion and attenuation by rock physics modeling. White proposed a model for seismic wave attenuation which is caused by the patchy saturation of two kinds of pore fluid [13]. The White model is extended afterward to be incorporated with Biot's theory [14] or to characterize the complex pore structure [15]. Chapman et al. [16] presented a model which considers the squirt flow related to cracks. This model is consistent with the Gassmann's relations at low frequency. Considering the anisotropy caused by the parallel arrangement of the cracks, Chapman [17] derived an anisotropic attenuation model. $\mathrm{Wu}$ et al. [18] gave three key assumptions: the model is consistent with the Gassmann-Wood prediction, partial 
saturation brings about higher attenuation value than full saturation, and the characteristic frequency on fluid mobility follows the theory of squirt flow. Based on these arguments, the Chapman model [16] is used to analyze the dispersion and attenuation of elastic parameters in the seismic frequency band in this paper, and the cracks are considered to be randomly arranged.

Rock physics plays an important role in reservoir prediction and can be used to obtain subsurface petrophysical properties $[19,20]$. The amplitude variation with offset (AVO) inversion is an effective method in reservoir interpretation and prediction [21-23]. The exact and approximate equations of Zoeppritz's equation are adopted for the AVO inversion [24-26]. The approximate equations have good linearity and clear physical meaning [27-30] and can be more effectively applied to reservoir prediction. Exact Zoeppritz's equation is a nonlinear forward operator, which may increase computation cost, while it is more suitable for far offset information [31]. With the development of exploration and exploitation, the lithologic trap reservoir has drawn much attention, and accurate description for hydrocarbon-bearing rocks is required. It brings huge challenges to fluid discrimination. There are mainly two methods for fluid discrimination by incorporating rock physics and seismic inversion [32]. One is to predict reservoir petrophysical properties directly by using the empirical or theoretical rock physics model [33]. The other is to identify fluid indirectly by using elastic parameters $[34,35]$. The prediction precision of the former method is based on the accuracy of the rock physics model, which is the quantitative interpretation method. The latter depends on the understanding of the relationship between petrophysical properties and elastic parameters, which is the qualitative interpretation method. The AVO inversion shows a certain ability in lithology prediction and fluid discrimination $[36,37]$. However, the elastic parameter inversion based on Zoeppritz's equation does not consider the effect of dispersion and attenuation caused by pore fluid. Wilson et al. proposed frequencydependent AVO (FAVO) following the approximation of Smith and Gidlow [38]. And the dispersion term used for fluid discrimination can be obtained by using FAVO inversion. The resolution of spectral decomposition can affect the accuracy of FAVO inversion. Wu et al. [39] and Luo et al. [40] used the smooth pseudo WignerVille distribution and inverse spectral decomposition for the time-frequency analysis, respectively, to enhance the resolution and accuracy of FAVO inversion results. In order to identify the hydrocarbon-bearing reservoir accurately, more sensitive frequency-dependent fluid factor needs to be studied [41]. Zhang et al. proposed a new fluid factor following the approximation of Russell et al. [42]. Chen et al. [43] and Wang et al. [44] suggested P-wave difference dispersion factor (PDDF) and bulk modulus difference dispersion factor (MDDF) to identify pore fluid, respectively.

In this paper, the attenuation and dispersion of elastic parameters are analyzed in detail to identify which is more dispersion sensitive. The results show that the pore fluid can bring more attenuation to Russell fluid factor, Lamé parameter, and bulk modulus, compared with $\mathrm{P}$-wave velocity. This is critical for us to decide which dispersion term to be used in the FAVO inversion. In addition, the applicability of Russell AVO approximation is analyzed, and a more reasonable range of the pending parameter is given, which can avoid a large deviation. Then, based on FAVO inversion, the dispersion terms and PDDFs related to Russell fluid factor, Lamé parameter, and bulk modulus are derived. The PDDFs are superior to dispersion terms in identifying the gas-bearing reservoir. In order to improve the antinoise ability of the inversion, $\ell 1$ norm regularization term [45] is applied.

\section{Theory and Method}

The current study of fluid identification is based on the dispersion and attenuation of elastic parameters. Therefore, the Chapman model [16] is used to analyze which parameter is more sensitive to the dispersion and attenuation induced by the pore fluid. Then, the theories of sparse constrained inversion spectral decomposition and frequency-dependent AVO inversion are summarized. In this section, the formula of frequency-dependent AVO is rewritten with respect to other elastic parameters and the applicability of Russell AVO approximation is analyzed.

2.1. Dispersion and Attenuation Analysis of Elastic Parameters. Chapman et al. [16] presented a frequencydependent model which considers the wave-induced exchange of fluids between pores and cracks, as well as between cracks. The cracks in this model are randomly arranged without causing anisotropy. In the lowfrequency limit, this model is consistent with the predicted result by using the Gassmann equation. By correcting the isotropic elastic tensor of the material with the perturbation due to the presence of cracks and pores, it defines a valid expression:

$$
C_{i j k l}=C_{i j k l}^{0}(\lambda, \mu)-C_{i j k l}^{1}\left(\lambda, \mu, \omega, \tau, \phi, r, \varepsilon, \kappa_{f}\right),
$$

where $C_{i j k l}^{0}(\bullet)$ is the isotropic elastic tensor without inclusions and $C_{i j k l}^{1}(\bullet)$ is the term affected by cracks and pores; $\lambda, \mu, \omega, \tau, \phi, r, \varepsilon$, and $\kappa_{f}$ are the Lamé parameter, shear modulus, angular frequency, timescale parameter, porosity, crack aspect ratio, crack density, and fluid bulk modulus, respectively. The detailed expression of $C_{i j k l}$ is given by Chapman et al. [46].

According to equation (1), we can get the elastic parameters influenced by frequency and fluid. Following the arguments of Chapman et al., the S-wave velocity also has attenuation and dispersion. This is because some of the cracks will necessarily have an orientation such that they will be compressed due to applied shear stress [47]. The attenuation of S-wave velocity is smaller than that of P-wave velocity in the Chapman model. To obtain attenuation characteristics of different elastic parameters, 


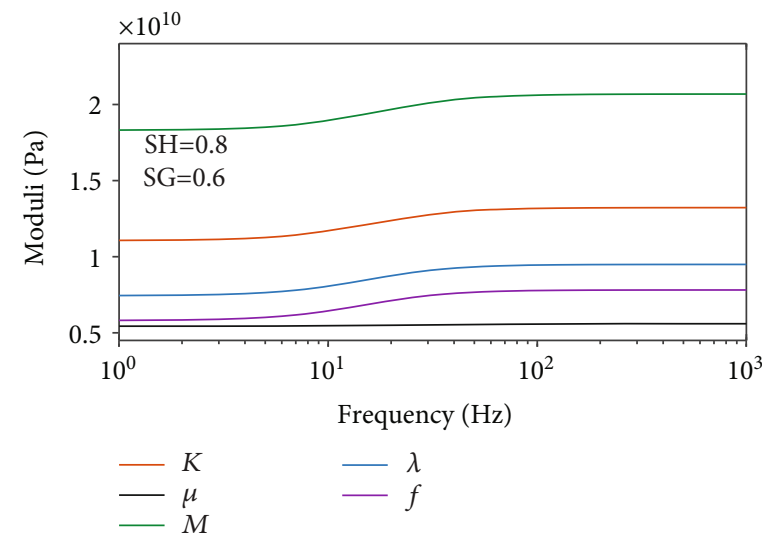

(a)

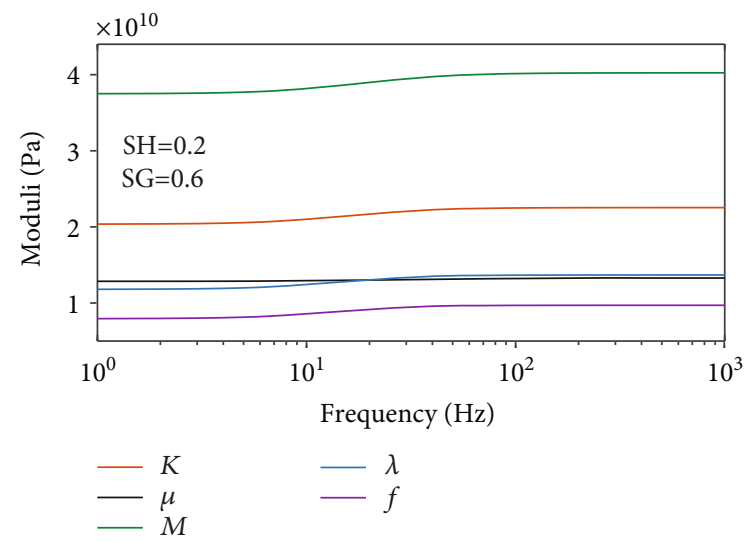

(c)

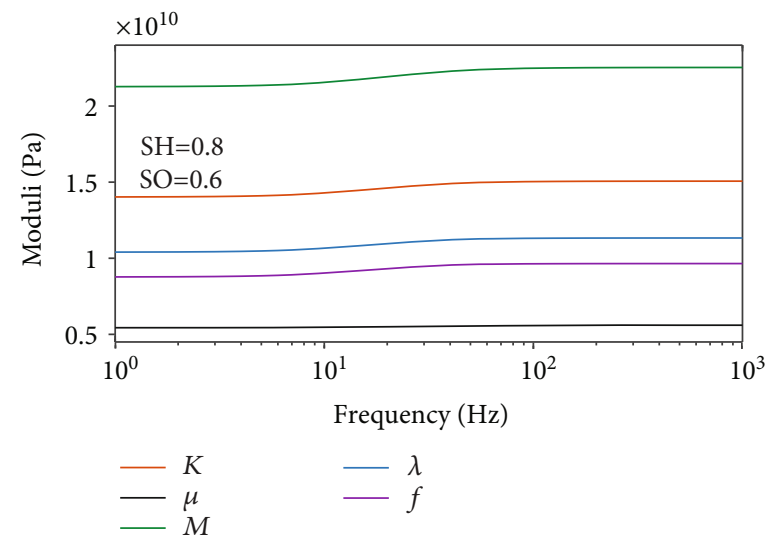

(b)

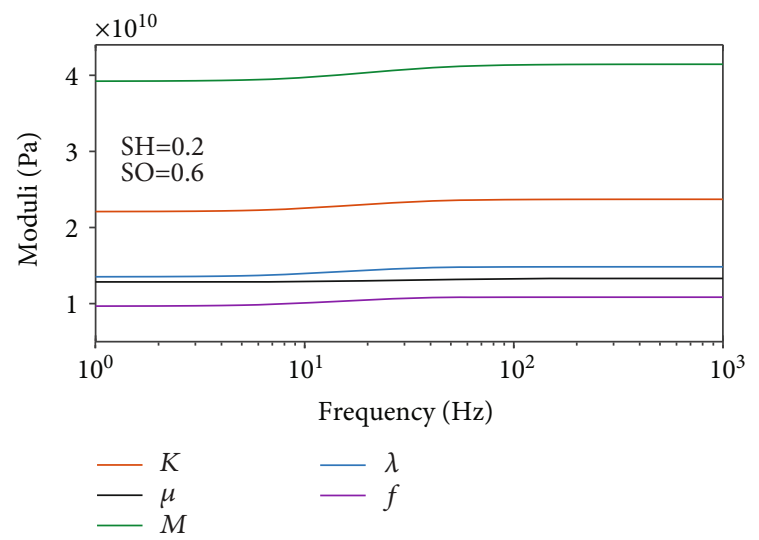

(d)

Figure 1: Dispersion analysis of different elastic parameters. SH indicates shale content, $\mathrm{SH}=0.2$ corresponds to sandstone reservoir and $\mathrm{SH}=0.8$ corresponds to shale reservoir; SG indicates gas saturation in gas-water mixed fluid and SO indicates oil saturation in oil-water mixed fluid; $K, \mu, M, \lambda$, and $f$ indicate the bulk modulus, shear modulus, $\mathrm{P}$-wave modulus, Lamé parameter, and Russell fluid factor, respectively; the value of $\gamma_{\text {dry }}^{2}$ is given as 2.3. (a) Gas-bearing shale reservoir; (b) oil-bearing shale reservoir; (c) gas-bearing sandstone reservoir; (d) oil-bearing sandstone reservoir.

other fluid-sensitive frequency-dependent elastic parameters are studied by removing the shear modulus from the P-wave modulus:

$$
\begin{aligned}
& \lambda=M-2 \mu, \\
& K=M-\frac{4}{3} \mu, \\
& f=M-\gamma_{\mathrm{dry}}^{2} \mu,
\end{aligned}
$$

where $K, M$, and $f$ are the bulk modulus, $\mathrm{P}$-wave modulus, and Russell fluid factor, respectively; $\gamma_{\text {dry }}^{2}$ is the weighting parameter associated with the dry rock and $\gamma_{\text {dry }}^{2}=\left(V_{P} / V_{S}\right)_{\text {dry }}^{2}[35] ; V_{P}$ indicates P-wave velocity, and $V_{S}$ indicates S-wave velocity; subscript dry indicates the dry rock. In the FAVO inversion, the dispersion terms which are induced by the pore fluid are related to these parameters. Thus, these parameters are analyzed before the introduction of FAVO inversion.
The dispersion characteristics of elastic parameters are shown in Figure 1. It shows that the shear modulus is affected by the frequency component quite weakly in both the sandstone $(\mathrm{SH}=0.2)$ and the shale $(\mathrm{SH}=0.8)$. For a particular lithology, the change of pore fluid has a great influence on Russell fluid factor $(f)$, Lamé parameter $(\lambda)$, bulk modulus $(K)$, and P-wave modulus $(M)$, instead of the shear modulus $(\mu)$. The dispersion brought by gas ( $\mathrm{SG}=0.6)$ is much larger than that of oil $(\mathrm{SO}=0.6)$. The value of $\gamma_{\mathrm{dry}}^{2}$ is discussed by Russell et al. [35]. We choose an intermediate value 2.3 of the values they listed. This parameter is also discussed later in the paper.

Figure 2 shows the attenuation of each elastic parameter under different reservoir petrophysical properties. The used parameter values of this model are summarized in Table 1. Since the dispersion and attenuation described by this rock physics model are caused by the effect of the pore fluid, the value of attenuation here corresponds to the dispersion magnitude. Figure 2 indicates that the attenuation values of Russell fluid factor, Lamé parameter $(\lambda)$, bulk modulus $(K)$, P-wave modulus $(M)$, and shear modulus $(\mu)$ of the 


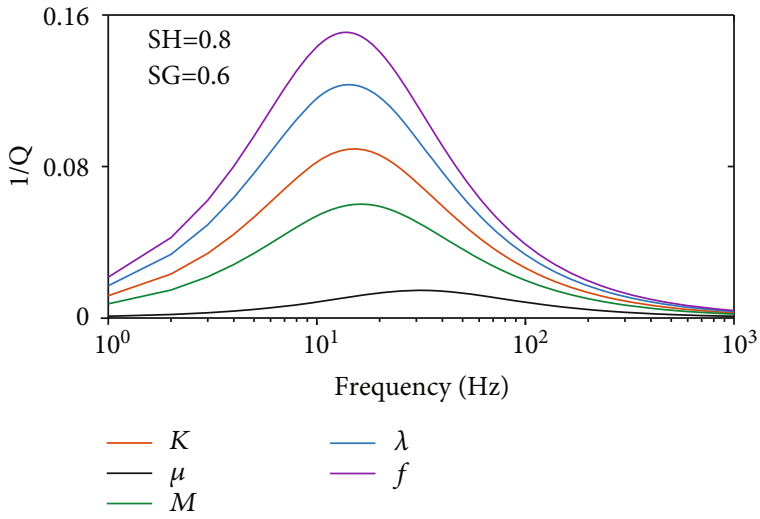

(a)

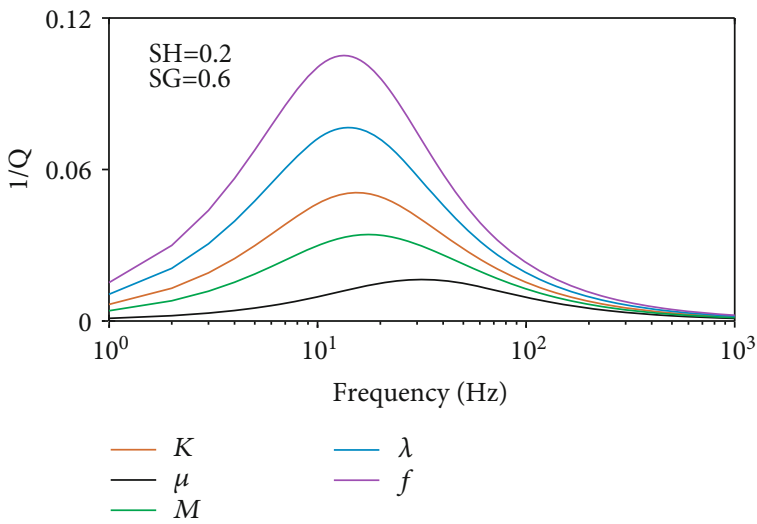

(c)

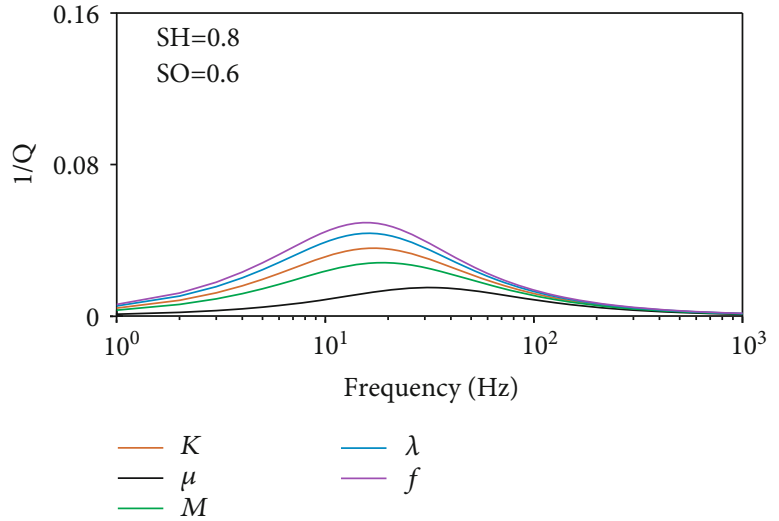

(b)

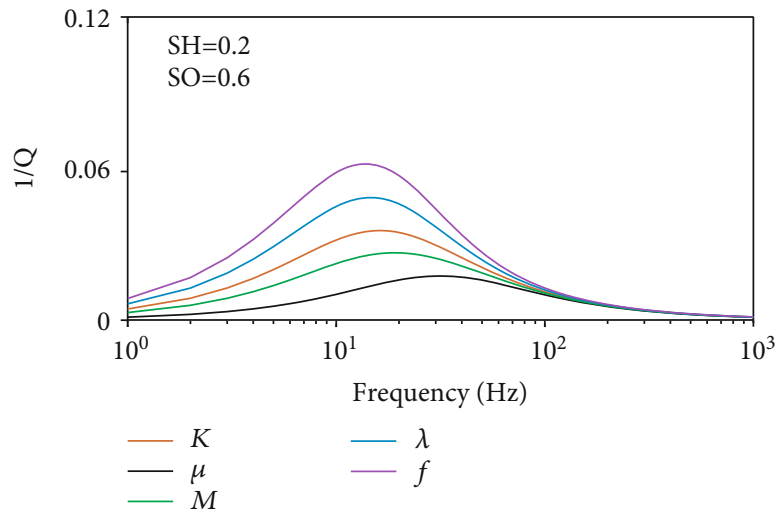

(d)

Figure 2: Attenuation analysis of different elastic parameters. SH indicates shale content, $\mathrm{SH}=0.2$ corresponds to sandstone reservoir and $\mathrm{SH}=0.8$ corresponds to shale reservoir; SG indicates gas saturation in gas-water mixed fluid and SO indicates oil saturation in oil-water mixed fluid; $K, \mu, M, \lambda$, and $f$ indicate the bulk modulus, shear modulus, $\mathrm{P}$-wave modulus, Lamé parameter, and Russell fluid factor, respectively; the value of $\gamma_{\mathrm{dry}}^{2}$ is given as 2.3. (a) Gas-bearing shale reservoir; (b) oil-bearing shale reservoir; (c) gas-bearing sandstone reservoir; (d) oil-bearing sandstone reservoir.

TABLE 1: The used parameter values in the Chapman model.

\begin{tabular}{|c|c|c|c|c|c|}
\hline Mineral/fluid & $K(\mathrm{GPa})$ & $\mu(\mathrm{GPa})$ & $\rho\left(\mathrm{g} / \mathrm{cm}^{3}\right)$ & Parameters & Value \\
\hline Sand & 37 & 44 & 2.65 & Time scale $(\tau)$ & $5 \times 10^{-3}$ \\
\hline Shale & 21 & 7 & 2.60 & Crack density $(\varepsilon)$ & 0.1 \\
\hline Water & 2.62 & 0 & 1.01 & Porosity $(\phi)$ & 0.25 \\
\hline Oil & 1.44 & 0 & 0.85 & \multirow{2}{*}{ Crack aspect ratio $(r)$} & \multirow{2}{*}{0.001} \\
\hline Gas & 0.05 & 0 & 0.21 & & \\
\hline
\end{tabular}

hydrocarbon-bearing reservoir are distributed from large to small. The elastic parameter attenuation value of the shale reservoir $(\mathrm{SH}=0.8)$ is larger than that of the sandstone reservoir $(\mathrm{SH}=0.2)$. The attenuation of the gas-bearing reservoir $(\mathrm{SG}=0.6)$ is larger than that of the oil-bearing reservoir $(\mathrm{SO}=0.6)$. It also shows that the attenuation of shear modulus is not affected by the pore fluid at low frequency. The attenuation of $\mathrm{P}$-wave modulus is the same as that of $\mathrm{P}$ wave velocity. Thus, the attenuation of Russell fluid factor, Lamé parameter, and bulk modulus is larger than that of $\mathrm{P}$ wave velocity and P-wave modulus. Finally, we can obtain the dispersion terms of Russell fluid factor, Lamé parameter, or bulk modulus to improve the precision of FAVO inversion.

\subsection{Sparse Constrained Inversion Spectral Decomposition. In} order to use the dispersion terms to identify the hydrocarbon-bearing rocks, the time-frequency distribution should be obtained as a prerequisite. According to the previous study [40, 44], inverse spectral decomposition has a higher time-frequency resolution. It has higher accuracy compared with other methods including short-time Fourier transform, wavelet transform, generalized $S$ transform and Wigner-Ville distribution. Spectral decomposition based on 
sparse constrained inversion is thus used to obtain the highresolution time-frequency distribution.

Using a dictionary of Ricker wavelets with different central frequencies, the seismic data can be decomposed into corresponding frequency-dependent pseudoreflectivity. The convolutional model can be represented by a linear system of equations [48], and the seismic trace s can be expressed as

$$
\mathbf{s}=\left(\begin{array}{llll}
\mathbf{W}_{1} & \mathbf{W}_{2} & \cdots & \mathbf{W}_{l}
\end{array}\right)\left(\begin{array}{c}
\mathbf{r}_{1} \\
\mathbf{r}_{2} \\
\vdots \\
\mathbf{r}_{l}
\end{array}\right)=\mathbf{R} \mathbf{m}
$$

where $\mathbf{W}_{i}$ refers to the Ricker wavelet with central frequency $f_{i}$ and $\mathbf{r}_{i}$ refers to the corresponding pseudoreflectivity sequence; $\mathbf{R}$ indicates the dictionary of Ricker wavelets and $\mathbf{m}$ indicates the vector containing the pseudoreflectivity sequences [40].

The $\ell 1$ norm regularization term can be applied to obtain a high-resolution result $[45,49]$ and it can promote a sparser solution than $\ell 2$ norm. The objective function $J$ can be established based on $\ell 1$ norm constraint as shown in equation (4). It can be solved by utilizing SPGL1 algorithm, which is used for one-norm regularized least squares and is suitable for problems that are large scale [50]:

$$
J=\|\mathbf{s}-\mathbf{R} \mathbf{m}\|^{2}+\zeta\|\mathbf{m}\|_{1}
$$

where $\zeta$ is the trade-off parameter which balances the sparsity and the misfit.

2.3. Frequency-Dependent AVO Inversion. FAVO is based on the approximation of Zoeppritz's equation [38, 39]. Aki and Richards [27] derived a simplified form of P-wave reflectivity in terms of density, P- and S-wave velocities. Gray et al. [51] provided two approximation equations about Lamé parameter, bulk modulus, shear modulus, and density. Another approximation considering fluid factor is derived by Russell et al. [35]. From the appendix, the approximate form proposed by Russell et al. has good general applicability, which can reduce to other approximations by modifying the parameter $\gamma_{\text {dry }}^{2}$.

Figure 3 illustrates the AVO features of these four AVO approximations and the value of $\gamma_{\text {dry }}^{2}$ is given as 2.3. The relevant upper and lower elastic parameters of the interface are shown in Table 2. Although they have different expressions, the AVO results are almost identical. Therefore, the FAVO study conducted below is based on the same accuracy of different AVO approximations. Since $\gamma_{\text {dry }}^{2}$ is usually not available, it should be set to a fixed value by users. Equation (A.6) will cause a large deviation when the value of $\gamma_{\mathrm{dry}}^{2}$ is very close to $\left(V_{P} / V_{S}\right)_{\text {sat }}^{2}$, which implies that there is no fluid component in the rock. According to Table 2, the value of $\left(V_{P} / V_{S}\right)_{\text {sat }}^{2}$ is 2.6213 . Figure 4 shows that if the value of $\gamma_{\text {dry }}^{2}$ is very close to 2.6213, the AVO curve deviates from the

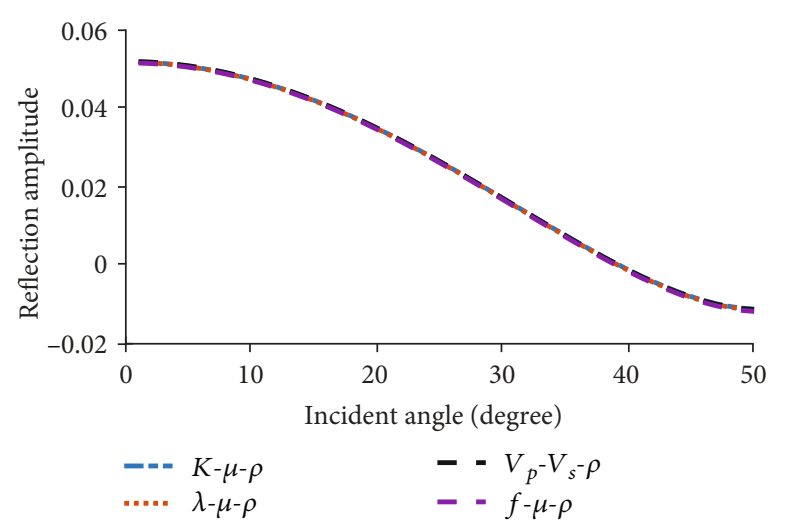

FIGURE 3: Reflection amplitude analysis with incident angle of different AVO approximations. $V_{P^{-}}-V_{S^{-}} \rho$ indicates the AkiRichards AVO approximation as equation (A.1) shows; $\lambda-\mu-\rho$ indicates the AVO approximation as equation (A.4) shows; $K-\mu-\rho$ indicates the AVO approximation as equation (A.5) shows; $f-\mu-\rho$ indicates the Russell et al. AVO approximation as equation (A.6) shows.

TABle 2: Parameters of the upper and lower layers.

\begin{tabular}{lccc}
\hline & $\begin{array}{c}\text { P-wave } \\
\text { velocity }(\mathrm{m} / \mathrm{s})\end{array}$ & $\begin{array}{c}\text { S-wave } \\
\text { velocity }(\mathrm{m} / \mathrm{s})\end{array}$ & Density $\left(\mathrm{g} / \mathrm{cm}^{3}\right)$ \\
\hline Upper layer & 3300 & 2000 & 2.2 \\
Lower layer & 3500 & 2200 & 2.3 \\
\hline
\end{tabular}

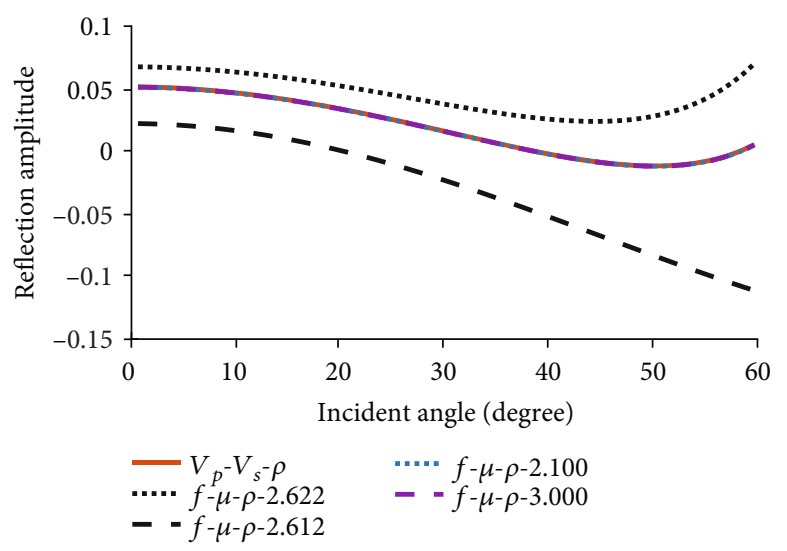

FIgURE 4: Reflection amplitude analysis with incident angle of different $\gamma_{\text {dry }}^{2} . \quad V_{P^{-}}-V_{S}-\rho$ indicates the Aki-Richards AVO approximation; $f-\mu-\rho-2.622, f-\mu-\rho-2.612, f-\mu-\rho-2.100$, and $f-\mu-\rho-$ 3.000 correspond to the Russell et al. AVO approximation with $\gamma_{\text {dry }}^{2}$ equaling to $2.622,2.612,2.100$, and 3.000 , respectively.

curve of Aki-Richards AVO approximation $(f-\mu-\rho-2.622$ and $f-\mu-\rho-2.612)$. When the values of $\gamma_{\text {dry }}^{2}$ and $\left(V_{P} / V_{S}\right)_{\text {sat }}^{2}$ are not close, as shown in Figure 4 , no matter the value of $\gamma_{\text {dry }}^{2}$ is smaller or larger than $\left(V_{P} / V_{S}\right)_{\text {sat }}^{2}$, the AVO curve can be consistent with that of Aki-Richards AVO approximation $(f-\mu-\rho-2.100$ and $f-\mu-\rho-3.000)$. Therefore, we need to ensure that the value of $\gamma_{\text {dry }}^{2}$ cannot be chosen as any possible value of $\left(V_{P} / V_{S}\right)_{\text {sat }}^{2}$ in the reservoir during the AVO inversion to prevent the values of $\gamma_{\text {dry }}^{2}$ and $\left(V_{P} / V_{S}\right)_{\text {sat }}^{2}$ from 
being very close. Since Poisson's ratio of rocks must be larger than 0 and $\left(V_{P} / V_{S}\right)_{\text {sat }}^{2}$ must be larger than 2 , therefore, equations (A.1), (A.4), and (A.5) are applicable to all the reservoirs without this deviation. An alternative way to avoid this deviation is to obtain all the potential values of $\left(V_{P} / V_{S}\right)_{\text {sat }}^{2}$ from the well logging data or the laboratory measurement, and then, take a value that is smaller or larger than them. However, by the application analysis of dispersion term later, only the smaller value is reasonable.

Wilson et al. derived a frequency-dependent AVO formula following the approximation of Smith and Gidlow [38]. This approximation eliminates the density term by using the Gardner formula. The density is not affected by the frequency, and the velocity is reversed. Thus, it is not recommended to use the Gardner formula in frequencydependent AVO inversion. Other AVO approximations containing density term, such as equations (A.1), (A.4), (A.5), and (A.6), are utilized here. Besides, the dispersion term of Russell fluid factor has been studied by some researchers [42]. These AVO approximations can be rewritten in a uniform formula:

$$
R_{P P}(\theta)=A(\theta) \frac{\Delta X}{X}+B(\theta) \frac{\Delta Y}{Y}+C(\theta) \frac{\Delta \rho}{\rho},
$$

where $A(\theta), B(\theta)$, and $C(\theta)$ are coefficients related to $\theta$; $\Delta X / X$ and $\Delta Y / Y$ are elastic parameter reflectivity of $X$ and $Y$, respectively.

Following the arguments of Wilson, we allow $X$ and $Y$ to vary with frequency $\omega$ :

$$
R_{P P}(\theta, \omega)=A(\theta) \frac{\Delta X}{X}(\omega)+B(\theta) \frac{\Delta Y}{Y}(\omega)+C(\theta) \frac{\Delta \rho}{\rho} .
$$

Due to the narrow frequency band in seismic data, this equation can be expanded as a Taylor series around a representative frequency $\omega_{0}$ :

$$
R_{P P}(\theta, \omega)-R_{P P}\left(\theta, \omega_{0}\right)=\left(\omega-\omega_{0}\right) A(\theta) I_{a}+\left(\omega-\omega_{0}\right) B(\theta) I_{b}
$$

where $I_{a}=d / d \omega(\Delta X / X)$ and $I_{b}=d / d \omega(\Delta Y / Y)$.

By using sparse constrained inversion spectral decomposition, the time-frequency spectrum $S(t, \theta, \omega)$ of seismic data $D(t, \theta)$ can be obtained. The spectral decomposition result $S$ can be affected by the overprint of source wavelet [52]. Therefore, spectral balancing should be performed on the spectral amplitude [53] to obtain the true spectral behaviour coming from the effect of geology and saturating fluid [18] by designing a suitable weight function $w(\omega)$ :

$$
B_{R}(t, \theta, \omega)=S(t, \theta, \omega) w(\omega) .
$$

According to $\mathrm{Wu}$ et al. [18], $w(\omega)$ is given as follows:

$$
w(\omega)=\frac{\sqrt{\sum_{k} S^{2}\left(t, \theta, \omega_{\mathrm{dom}}\right)}}{\sqrt{\sum_{k} S^{2}(t, \theta, \omega)}},
$$

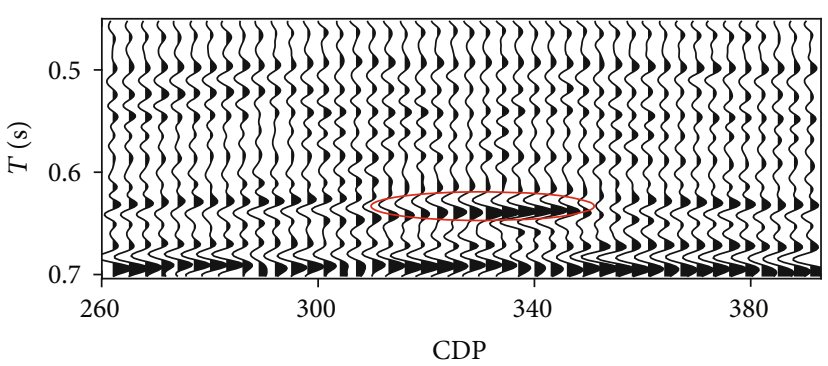

FIgURE 5: Stack section of a field dataset from HampsonRussell software. The seismic traces are shown with the intervals of 3 . The red circle highlights the location of the gas reservoir.

where $\omega_{\text {dom }}$ denotes the dominant frequency of the seismic data; $k$ is the number of sampling points in a defined window.

For the sample point $t_{0}$, the dispersion terms $I_{a}$ and $I_{b}$ can be obtained by solving the following equations:

$$
\begin{aligned}
B_{R}\left(t_{0}, \theta, \omega\right)- & R_{P P}\left(t_{0}, \theta, \omega_{\mathrm{dom}}\right)=\left(\omega-\omega_{\mathrm{dom}}\right) A\left(t_{0}, \theta\right) I_{a} \\
+ & \left(\omega-\omega_{\mathrm{dom}}\right) B\left(t_{0}, \theta\right) I_{b}, \\
R_{P P}\left(t_{0}, \theta, \omega_{\mathrm{dom}}\right)= & A\left(t_{0}, \theta\right) \frac{\Delta X}{X}\left(t_{0}, \omega_{\mathrm{dom}}\right) \\
& +B\left(t_{0}, \theta\right) \frac{\Delta Y}{Y}\left(t_{0}, \omega_{\mathrm{dom}}\right)+C\left(t_{0}, \theta\right) \frac{\Delta \rho}{\rho} .
\end{aligned}
$$

A simplified matrix form can be characterized by

$$
\mathbf{T}=\mathbf{D}\left[\begin{array}{c}
I_{a} \\
I_{b}
\end{array}\right]
$$

The matrices $\mathbf{T}$ and $\mathbf{D}$ are given as follows:

$$
\begin{aligned}
& \mathbf{T}=\left[\begin{array}{c}
B_{R}\left(t_{0}, \theta_{1}, \omega_{1}\right)-R_{P P}\left(t_{0}, \theta_{1}, \omega_{\mathrm{dom}}\right) \\
\vdots \\
B_{R}\left(t_{0}, \theta_{1}, \omega_{m}\right)-R_{P P}\left(t_{0}, \theta_{1}, \omega_{\mathrm{dom}}\right) \\
\vdots \\
B_{R}\left(t_{0}, \theta_{n}, \omega_{1}\right)-R_{P P}\left(t_{0}, \theta_{n}, \omega_{\mathrm{dom}}\right) \\
\vdots \\
B_{R}\left(t_{0}, \theta_{n}, \omega_{m}\right)-R_{P P}\left(t_{0}, \theta_{n}, \omega_{\mathrm{dom}}\right)
\end{array}\right], \\
& \mathbf{D}=\left[\begin{array}{cc}
\left(\omega_{1}-\omega_{\mathrm{dom}}\right) A\left(t_{0}, \theta_{1}\right) & \left(\omega_{1}-\omega_{\mathrm{dom}}\right) B\left(t_{0}, \theta_{1}\right) \\
\vdots & \vdots \\
\left(\omega_{m}-\omega_{\mathrm{dom}}\right) A\left(t_{0}, \theta_{1}\right) & \left(\omega_{m}-\omega_{\mathrm{dom}}\right) B\left(t_{0}, \theta_{1}\right) \\
\vdots & \vdots \\
\left(\omega_{1}-\omega_{\mathrm{dom}}\right) A\left(t_{0}, \theta_{n}\right) & \left(\omega_{1}-\omega_{\mathrm{dom}}\right) B\left(t_{0}, \theta_{n}\right) \\
\vdots & \vdots \\
\left(\omega_{m}-\omega_{\mathrm{dom}}\right) A\left(t_{0}, \theta_{n}\right) & \left(\omega_{m}-\omega_{\mathrm{dom}}\right) B\left(t_{0}, \theta_{n}\right)
\end{array}\right],
\end{aligned}
$$




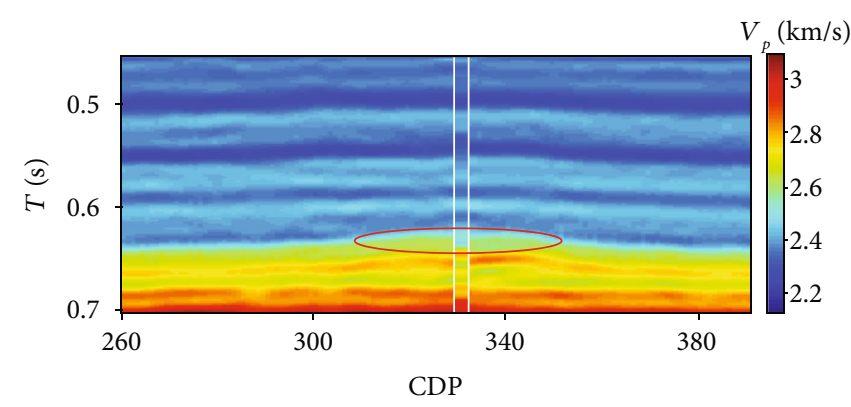

(a)

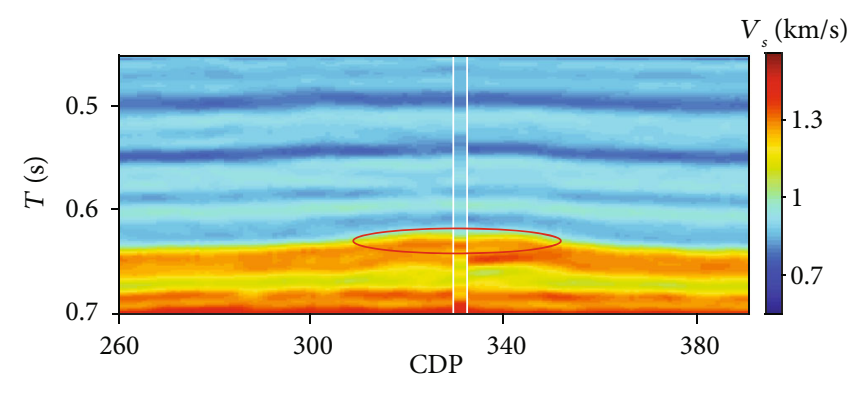

(b)

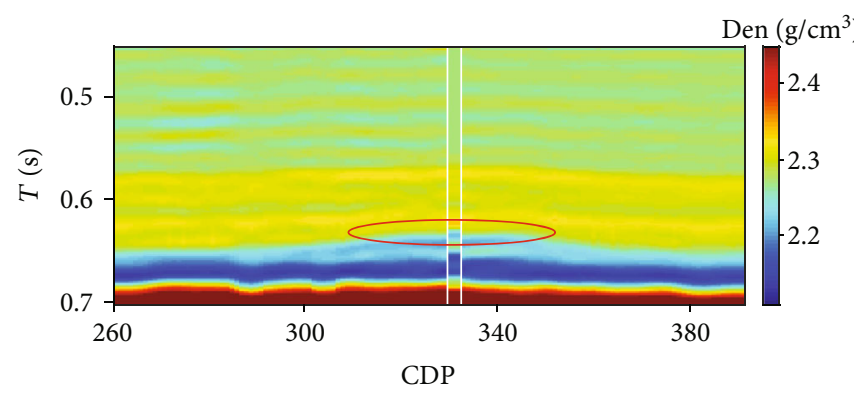

(c)

FIGURE 6: Inversion results based on the exact Zoeppritz's equation. (a) is the section of P-wave velocity, (b) is the section of S-wave velocity, and (c) is the section of density. The real well logs are inserted at CDP 330. The red circle highlights the location of the gas reservoir.

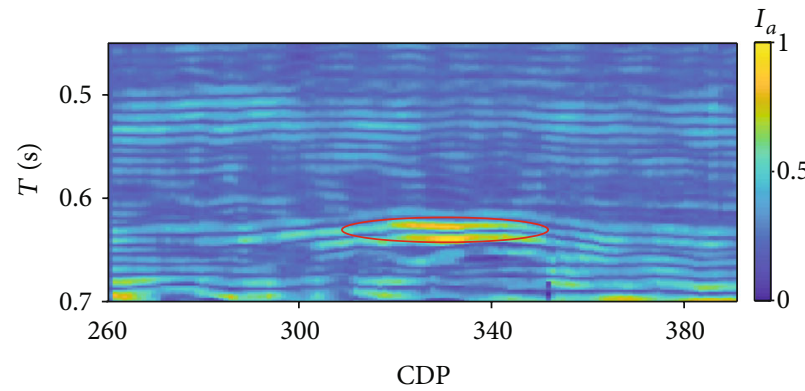

(a)

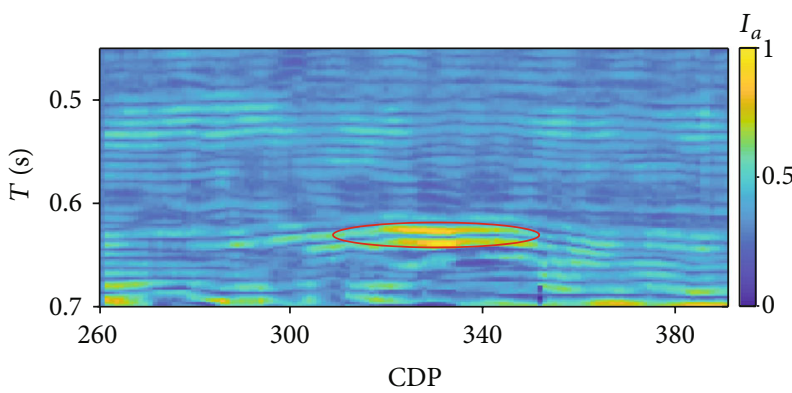

(c)

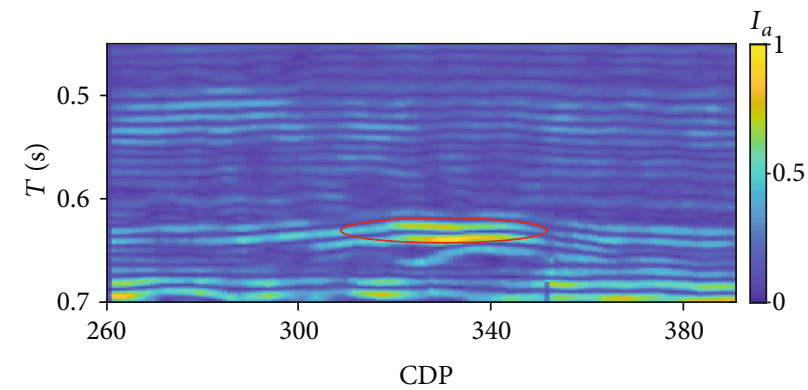

(b)

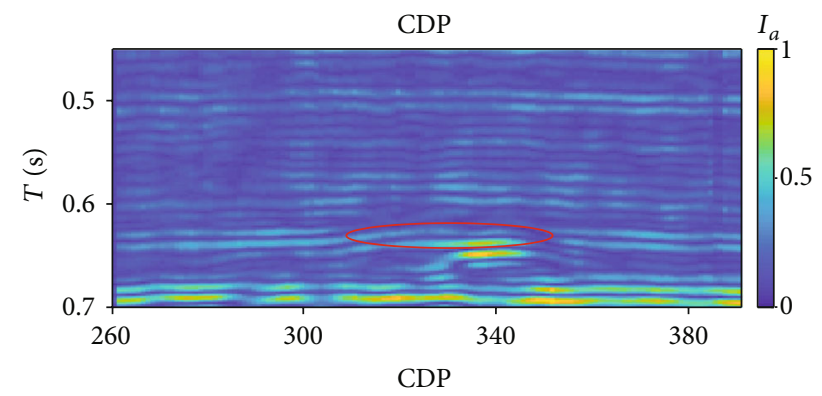

(d)

Figure 7: The results of FAVO inversion. $I_{a}$ indicates the dispersion term. (a) is the section of dispersion term of Lamé parameter, (b) is the section of dispersion term of bulk modulus, (c) is the section of dispersion term of Russell fluid factor, and (d) is the section of dispersion term of P-wave velocity. The red circle highlights the location of the gas reservoir. The dispersion terms are normalized. 
where $n$ is the number of angle gathers and $m$ is the number of frequencies after the spectral decomposition.

\section{Application}

The used field seismic data set is from HampsonRussell software, recorded over the shallow Cretaceous. Figure 5 shows the stack section with gathers from CDP 260 to 390. The angles vary from $3^{\circ}$ to $24^{\circ}$. The dominant frequency is about $35 \mathrm{~Hz}$. The red circle in Figure 5 indicates the location of gas reservoir with the strong reflector. A real gas well is located at CDP 330. From the well logging data, the lithology of the target reservoir is gas-bearing sandstone and the gas saturation is about $50 \%$. This is a typical gas reservoir, which can be used to test whether our method is valid or not. This set of data has been used as an application study by other researchers [54].

In order to obtain $\left(V_{S} / V_{P}\right)_{\text {sat }}^{2}$ in the FAVO inversion, the $\mathrm{P}$ - and $\mathrm{S}$-wave velocities are estimated based on the exact Zoeppritz's equation. The Bayesian framework is used to improve the stability of the inversion. For simplicity, $\left(V_{S} / V_{P}\right)_{\text {sat }}^{2}$ can be approximated to a fixed value with some accuracy loss. Figure 6 shows the inversion results. It shows that the accuracy of P-wave velocity around the red circle is suppressed due to the existence of the gas reservoir compared with the overlying strata. Figure 7(d) shows relatively weaker dispersion of P-wave velocity in the gas reservoir. There is also a deviation in the position of the strong dispersion which cannot match the reservoir location very well. Figures 7(a)$7(\mathrm{c})\left(\gamma_{\mathrm{dry}}^{2}=2.3\right)$ have the same dispersion characteristics and can better identify the fluid. The dispersion of Figure 7(b) is slightly weaker than that of Figures 7(a) and 7 (c). Figure 7 shows that the dispersion terms related to Russell fluid factor, Lamé parameter, and bulk modulus are superior to that of P-wave velocity, and among them, Russell fluid factor is slightly more prominent, followed by Lamé parameter.

The value of $\gamma_{\text {dry }}^{2}$ is determined from the well logging data. Figure 8 shows that all values of $\left(V_{P} / V_{S}\right)_{\text {sat }}^{2}$ are larger than 2.3. If we let $\gamma_{\mathrm{dry}}^{2}=4.1$ or 8.9 , the dispersion term of Russell fluid factor is unsatisfactory (Figures 9(a) and 9(b)). These two values of $\gamma_{\mathrm{dry}}^{2}$ are within the range of $\left(V_{P} / V_{S}\right)_{\text {sat }}^{2}$, and they can cause big deviations to the dispersion term. According to the previous analysis, the AVO approximation is accurate when the value of $\gamma_{\text {dry }}^{2}$ is much larger than $\left(V_{P} / V_{S}\right)_{\text {sat }}^{2}$. However, this does not mean that the dispersion term is correct. Figure 9(c) shows poor dispersion term even for a large value of $\gamma_{\mathrm{dry}}^{2}\left(\gamma_{\mathrm{dry}}^{2}=15\right)$. Therefore, $\gamma_{\mathrm{dry}}^{2}$ should be smaller than any possibility of $\left(V_{P} / V_{S}\right)_{\text {sat. }}^{2}$. In addition, from equations (2), if the value of $\gamma_{\mathrm{dry}}^{2}$ is $2,4 / 3$, or 0 , Russell fluid factor $f$ can be changed into Lamé parameter $\lambda$, bulk modulus $K$, or P-wave modulus $M$. As depicted in Figure 2, with a larger value of $\gamma_{\mathrm{dry}}^{2}$, the Russell fluid factor can have more attenuation induced by the pore fluid.

According to Chen et al. [43], we can derive new P-wave difference dispersion factor (PDDF) combining with the

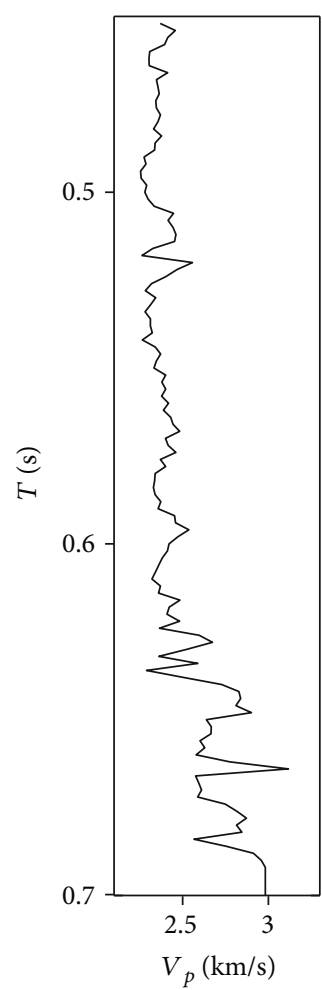

(a)

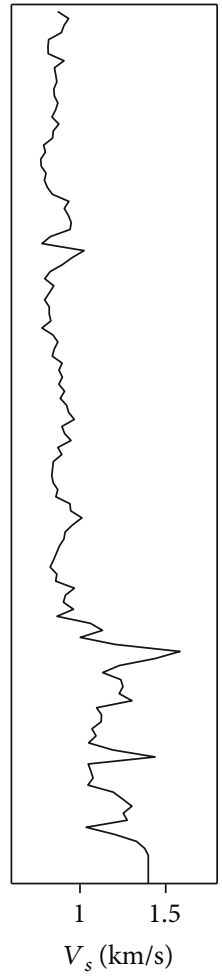

(b)

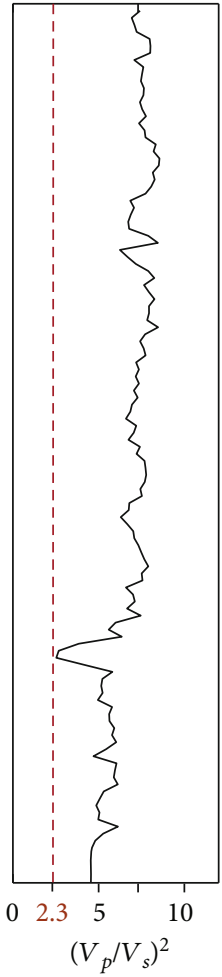

(c)
FIgURE 8: Well logging curves. (a) indicates P-wave velocity; (b) indicates S-wave velocity; (c) indicates $\left(V_{P} / V_{S}\right)_{\text {sat }}^{2}$.

P-wave difference. The new PDDFs are related to Russell fluid factor, Lamé parameter, and bulk modulus by replacing $I_{a}$ in the following equation:

$$
\operatorname{PDDF}=\Delta V_{P} \cdot I_{a},
$$

where $\Delta V_{P}$ is obtained by subtracting the $\mathrm{P}$-wave velocity of the upper layer from that of the lower layer. Figure 10 shows the prediction process of dispersion term and Pwave difference dispersion factor.

Figure 11 indicates that the PDDF is superior to dispersion term $I_{a}$ (Figure 7) in fluid discrimination. The section of PDDF has better accuracy and better continuity. Due to the difference in dispersion terms, the PDDF results are different obviously. The PDDFs related to Russell fluid factor, Lamé parameter, and bulk modulus are almost similar, and they are all better than that of P-wave velocity in identifying the gas-bearing rocks. By comparison, Russell fluid factor is slightly more obvious.

The various parameter curves at borehole-side trace are displayed in Figure 12. In Figure 12, there are two peaks on the curves of dispersion term and PDDF at the location of gas reservoir. It is consistent with the analysis of HampsonRussell software about the two sets of gas layer: top gas and base gas. The dispersion terms and PDDFs related to Russell fluid factor $(f)$, Lamé parameter $(\lambda)$, and bulk modulus $(K)$ are more pronounced. Due to the attenuation and dispersion caused by the gas reservoir, the inverted $\mathrm{P}$-wave velocity (Figure 12(b)) based on the elastic theory of Zoeppritz's 


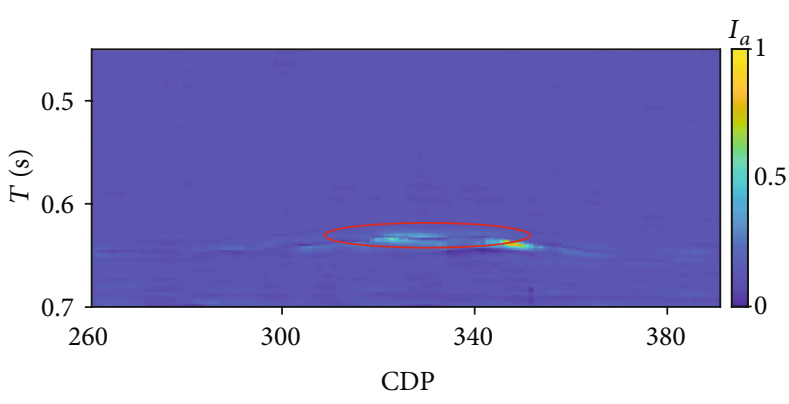

(a)

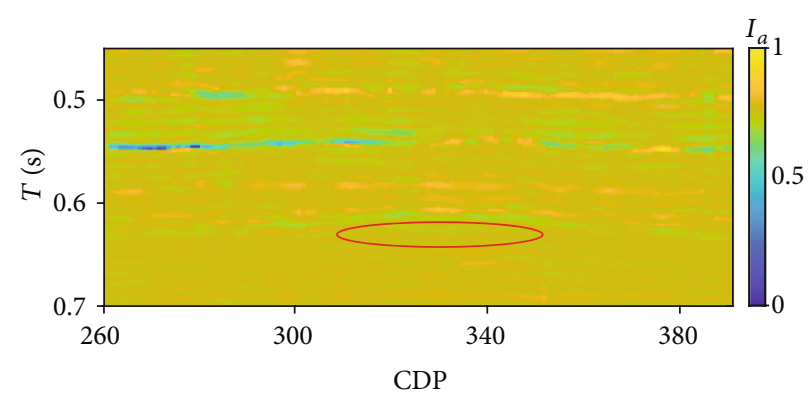

(b)

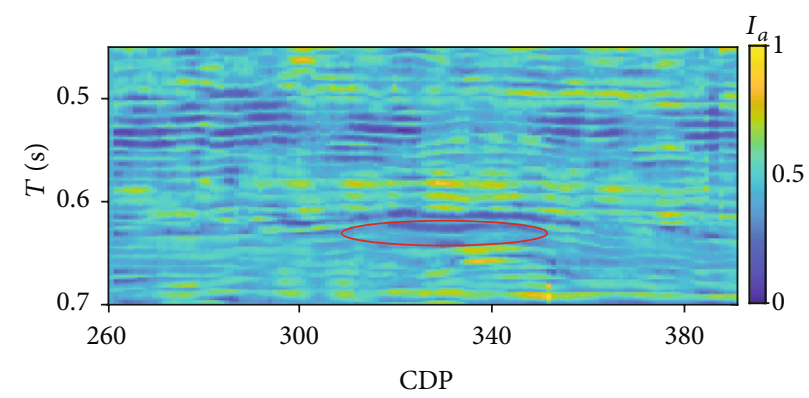

(c)

FIGURE 9: Analysis of dispersion terms of Russell fluid factor with different $\gamma_{\mathrm{dry}}^{2}$. (a) corresponds to the dispersion term with $\gamma_{\mathrm{dry}}^{2}=4.1$; (b) corresponds to the dispersion term with $\gamma_{\mathrm{dry}}^{2}=8.9$; (c) corresponds to the dispersion term with $\gamma_{\mathrm{dry}}^{2}=15$; the red circle highlights the location of the gas reservoir.

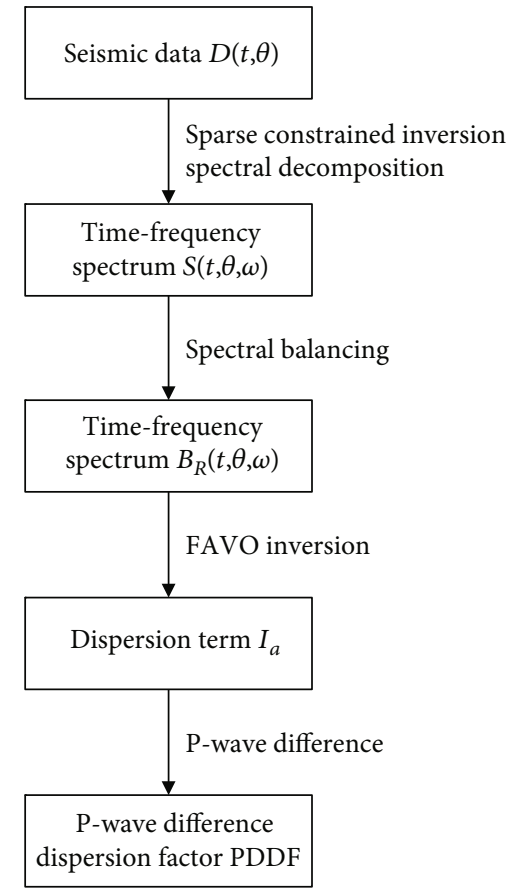

FIGURE 10: The prediction process of dispersion term and P-wave difference dispersion factor.

equation is affected and the prediction accuracy of the lower layer is unsatisfactory. This also explains why FAVO inversion can be used to identify oil and gas.

\section{Discussion and Conclusions}

Pore fluid can cause dispersion and attenuation of elastic parameters in the seismic frequency range. Therefore, the study of dispersion term of elastic parameter can be beneficial for fluid discrimination. By analysis, the shear modulus is almost unaffected by the pore fluid; however, the dispersion and attenuation of Russell fluid factor, Lamé parameter, and bulk modulus are stronger and decrease in turn. Uniform expression of dispersion terms of these parameters is derived, and $\mathrm{P}$-wave difference dispersion factors are studied by incorporating with P-wave difference.

The applicability of Russell AVO approximation is analyzed, and an inappropriate weighting parameter associated with the dry rock can cause a large deviation. In order to ensure that the AVO attribute and the dispersion term are correct and reasonable, the value $\gamma_{\text {dry }}^{2}$ should be smaller than any possibility of $\left(V_{P} / V_{S}\right)_{\text {sat }}^{2}$ from the well logging data or the laboratory measurement.

Field data application shows that the dispersion terms related to Russell fluid factor with proper weighting parameter, Lamé parameter, and bulk modulus are preferable, and among them, Russell fluid factor is slightly more prominent, followed by Lamé parameter, which is consistent with the Chapman model. The P-wave difference dispersion factors related to Russell fluid factor, Lamé parameter, and bulk modulus are almost similar and better than those of $\mathrm{P}$-wave velocity, and Russell fluid factor has a slight advantage in identifying pore fluid. 


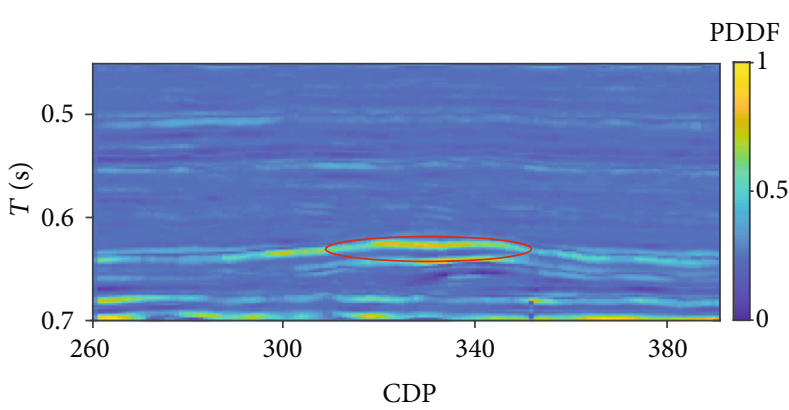

(a)

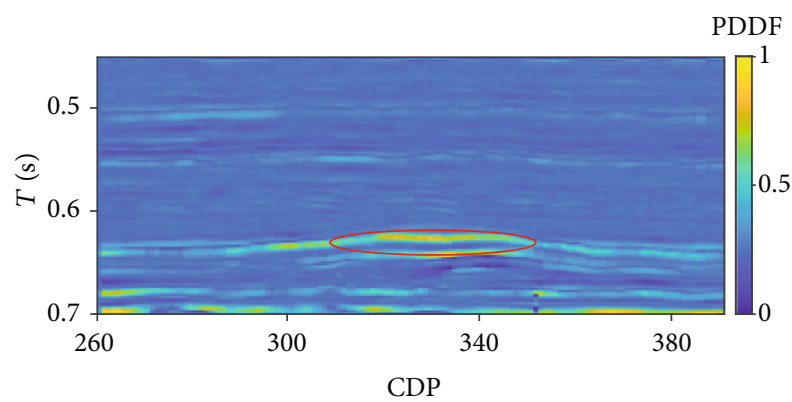

(c)

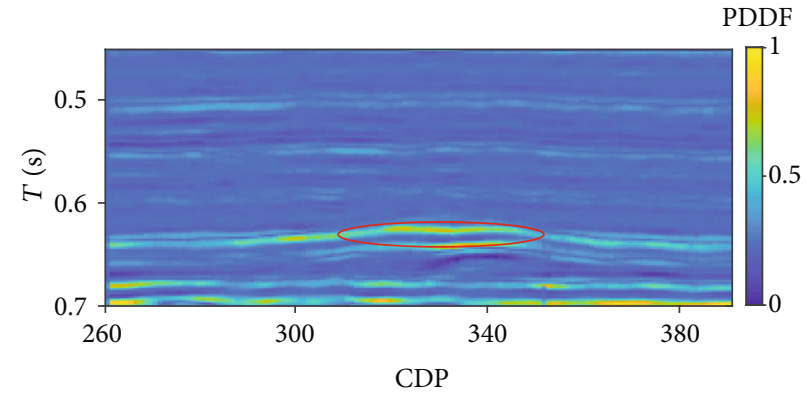

(b)

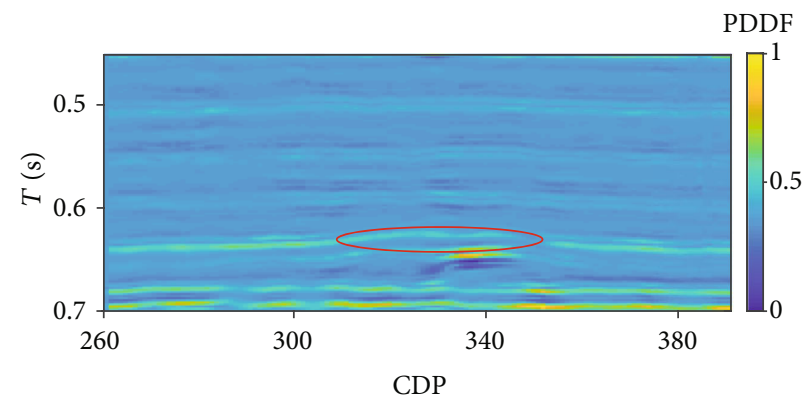

(d)

FIgUre 11: The P-wave difference dispersion factors of different dispersion terms. PDDF indicates the P-wave difference dispersion factor; (a) is the section of PDDF related to Lamé parameter, (b) is the section of PDDF related to bulk modulus, (c) is the section of PDDF related to Russell fluid factor $\left(\gamma_{\mathrm{dry}}^{2}=2.3\right)$, and $(\mathrm{d})$ is the section of PDDF related to P-wave velocity. The red circle highlights the location of the gas reservoir. The PDDFs are normalized.
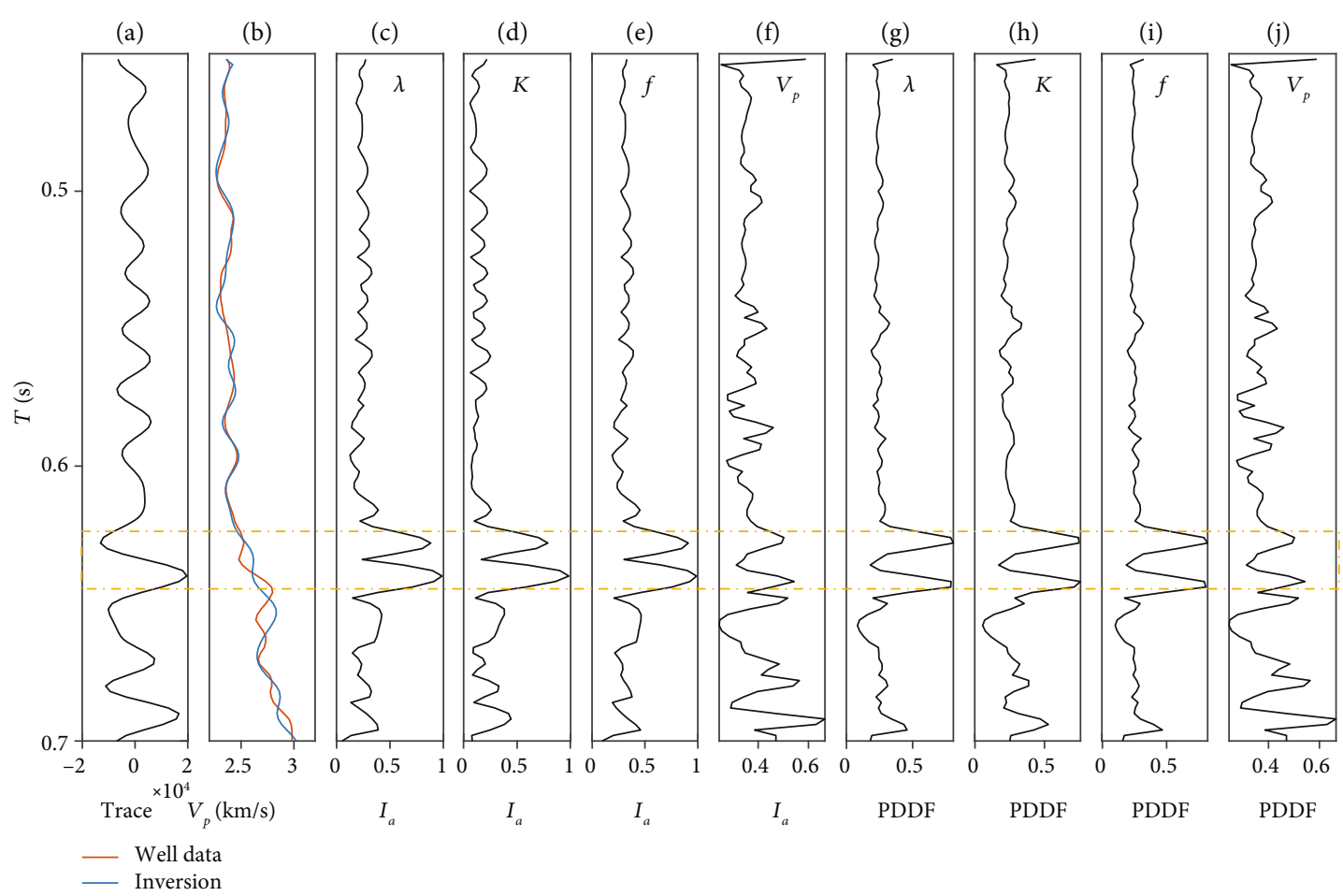

FIGURE 12: Display of various parameters at the well location. (a) is the poststack seismic trace; (b) is the P-wave velocity, the red curve is the real well log after smoothing, and the blue curve is the inverted result; (c) is the dispersion term of Lamé parameter $(\lambda)$; (d) is the dispersion term of bulk modulus $(K)$; (e) is the dispersion term of Russell fluid factor $(f)\left(\gamma_{\mathrm{dry}}^{2}=2.3\right)$; (f) is the dispersion term of P-wave velocity $\left(V_{P}\right)$; $(\mathrm{g})$ is the PDDF of Lamé parameter $(\lambda)$; (h) is the PDDF of bulk modulus $(K)$; (i) is the PDDF of Russell fluid factor $(f)\left(\gamma_{\text {dry }}^{2}=2.3\right)$; $(\mathrm{j})$ is the PDDF of P-wave velocity $\left(V_{P}\right)$. The yellow dotted rectangle highlights the location of the gas reservoir. 


\section{Appendix}

\section{The Relationship of Some Approximations of Zoeppritz's Equation}

Aki and Richards [27] derived a simplified form of P-wave reflectivity in terms of density and $\mathrm{P}$ - and $\mathrm{S}$-wave velocities as follows:

$$
\begin{aligned}
R_{P P}(\theta)= & \frac{1}{2} \sec ^{2} \theta \frac{\Delta V_{P}}{V_{P}}-4 \sin ^{2} \theta\left(\frac{V_{S}}{V_{P}}\right)^{2} \frac{\Delta V_{S}}{V_{S}} \\
& +\frac{1}{2}\left(1-4 \sin ^{2} \theta\left(\frac{V_{S}}{V_{P}}\right)^{2}\right) \frac{\Delta \rho}{\rho},
\end{aligned}
$$

where $\theta$ is the incident angle; $V_{P}, V_{S}$, and $\rho$ are the P-wave velocity, S-wave velocity, and density of the fluid-filled rock, respectively; $\Delta V_{P} / V_{P}, \Delta V_{S} / V_{S}$, and $\Delta \rho / \rho$ are the P-wave velocity reflectivity, $\mathrm{S}$-wave velocity reflectivity, and density reflectivity, respectively; $\Delta V_{P}, \Delta V_{S}$, and $\Delta \rho$ are the differences between the lower and upper layers of the interface.

With the relationships between material properties [55],

$$
\begin{aligned}
& \frac{\Delta V_{P}}{V_{P}}=\frac{1}{2}\left(\frac{\Delta M}{M}-\frac{\Delta \rho}{\rho}\right), \\
& \frac{\Delta V_{S}}{V_{S}}=\frac{1}{2}\left(\frac{\Delta \mu}{\mu}-\frac{\Delta \rho}{\rho}\right),
\end{aligned}
$$

and the relationships between $\mathrm{P}$-wave modulus, S-wave modulus, bulk modulus, and Lamé parameter summarized as follows,

$$
\Delta M=\Delta K+\frac{4}{3} \Delta \mu=\Delta \lambda+2 \Delta \mu
$$

another two approximation equations can be derived as follows, which are the same as the equations given by Gray et al. [51]:

$$
\begin{aligned}
R_{P P}(\theta)= & \left(\frac{1}{4}-\frac{1}{2}\left(\frac{V_{S}}{V_{P}}\right)_{\text {sat }}^{2}\right) \sec ^{2} \theta \frac{\Delta \lambda}{\lambda} \\
& +\left(\frac{1}{2} \sec ^{2} \theta-2 \sin ^{2} \theta\right)\left(\frac{V_{S}}{V_{P}}\right)_{\text {sat }}^{2} \frac{\Delta \mu}{\mu} \\
& +\frac{1}{4}\left(1-\tan ^{2} \theta\right) \frac{\Delta \rho}{\rho}, \\
R_{P P}(\theta)= & \left(\frac{1}{4}-\frac{1}{3}\left(\frac{V_{S}}{V_{P}}\right)_{\operatorname{sat}}^{2}\right) \sec ^{2} \theta \frac{\Delta K}{K} \\
& +\left(\frac{1}{3} \sec ^{2} \theta-2 \sin ^{2} \theta\right)\left(\frac{V_{S}}{V_{P}}\right)_{\text {sat }}^{2} \frac{\Delta \mu}{\mu} \\
& +\frac{1}{4}\left(1-\tan ^{2} \theta\right) \frac{\Delta \rho}{\rho},
\end{aligned}
$$

where $\Delta M / M, \Delta \lambda / \lambda, \Delta K / K$, and $\Delta \mu / \mu$ are the P-wave modulus reflectivity, Lamé parameter reflectivity, bulk modulus reflectivity, and shear modulus reflectivity, respectively; subscript sat indicates the fluid-filled rock.

Russell et al. [35] derived a new AVO approximation, which has the same form as equations (A.4) and (A.5):

$$
\begin{aligned}
R_{P P}(\theta)= & \left(\frac{1}{4}-\frac{\gamma_{\text {dry }}^{2}}{4}\left(\frac{V_{S}}{V_{P}}\right)_{\text {sat }}^{2}\right) \sec ^{2} \theta \frac{\Delta f}{f} \\
& +\left(\frac{\gamma_{\text {dry }}^{2}}{4} \sec ^{2} \theta-2 \sin ^{2} \theta\right)\left(\frac{V_{S}}{V_{P}}\right)_{\text {sat }}^{2} \frac{\Delta \mu}{\mu} \\
& +\frac{1}{4}\left(1-\tan ^{2} \theta\right) \frac{\Delta \rho}{\rho} .
\end{aligned}
$$

If the value of $\gamma_{\mathrm{dry}}^{2}$ is 2 or $4 / 3$, equation (A.6) can reduce to equation (A.4) or (A.5). Besides, if the value of $\gamma_{\text {dry }}^{2}$ is 0 , equation (A.1) can be obtained by multiplying equation (A.6) by 2 .

\section{Data Availability}

All model data during this study are listed; researchers can replicate the analysis. But the real seismic data in the application section are not available because they involve business secrets.

\section{Conflicts of Interest}

The authors declare that they have no conflicts of interest.

\section{Acknowledgments}

The authors gratefully acknowledge financial supports from the National Natural Science Foundation of China (Nos. 41774131, 41774129, and 41874128), the Science Foundation of China University of Petroleum, Beijing (No. 2462018QZDX01), and the Young Elite Scientists Sponsorship Program by CAST (No. 2018QNRC001).

\section{References}

[1] G. Mavko, T. Mukerji, and J. Dvorkin, The Rock Physics Handbook: Tools for Seismic Analysis of Porous Media, Cambridge university press, 2009.

[2] G. T. Kuster and M. N. Toksöz, "Velocity and attenuation of seismic waves in two-phase media: part I. Theoretical formulations," Geophysics, vol. 39, no. 5, pp. 587-606, 1974.

[3] T. T. Wu, "The effect of inclusion shape on the elastic moduli of a two-phase material," International Journal of Solids and Structures, vol. 2, no. 1, pp. 1-8, 1966.

[4] J. G. Berryman, "Single-scattering approximations for coefficients in Biot's equations of poroelasticity," The Journal of the Acoustical Society of America, vol. 91, no. 2, pp. 551-571, 1992.

[5] M. A. Biot, "Theory of propagation of elastic waves in a fluidsaturated porous solid. I. Low-frequency range," The Journal of the Acoustical Society of America, vol. 28, no. 2, pp. 168-178, 1956. 
[6] L. Thomsen, "Biot-consistent elastic moduli of porous rocks: low-frequency limit," Geophysics, vol. 50, no. 12, pp. 27972807, 1985.

[7] Z. Wang and A. Nur, "Elastic wave velocities in porous media: a theoretical recipe," Seismic and Acoustic Velocities in Reservoir Rocks, vol. 2, pp. 1-35, 1992.

[8] R. G. Keys and S. Xu, "An approximation for the Xu-White velocity model," Geophysics, vol. 67, no. 5, pp. 1406-1414, 2002.

[9] F. Ruiz and A. Cheng, "A rock physics model for tight gas sand," The Leading Edge, vol. 29, no. 12, pp. 1484-1489, 2010.

[10] W. F. Murphy III, "Effects of partial water saturation on attenuation in Massilon sandstone and Vycor porous glass," The Journal of the Acoustical Society of America, vol. 71, no. 6, pp. 1458-1468, 1982.

[11] W. F. Murphy III, "Acoustic measures of partial gas saturation in tight sandstones," Journal of Geophysical Research: Solid Earth, vol. 89, no. B13, pp. 11549-11559, 1984.

[12] S. Nakagawa, T. J. Kneafsey, T. M. Daley, B. M. Freifeld, and E. V. Rees, "Laboratory seismic monitoring of supercritical $\mathrm{CO}_{2}$ flooding in sandstone cores using the Split Hopkinson Resonant Bar technique with concurrent X-ray computed tomography imaging," Geophysical Prospecting, vol. 61, no. 2, pp. 254-269, 2013.

[13] J. E. White, "Computed seismic speeds and attenuation in rocks with partial gas saturation," Geophysics, vol. 40, no. 2, pp. 224-232, 1975.

[14] N. C. Dutta and H. Odé, "Attenuation and dispersion of compressional waves in fluid-filled porous rocks with partial gas saturation (White model)-part I: Biot theory," Geophysics, vol. 44, no. 11, pp. 1777-1788, 1979.

[15] D. L. Johnson, "Theory of frequency dependent acoustics in patchy-saturated porous media," The Journal of the Acoustical Society of America, vol. 110, no. 2, pp. 682-694, 2001.

[16] M. Chapman, S. V. Zatsepin, and S. Crampin, "Derivation of a microstructural poroelastic model," Geophysical Journal International, vol. 151, no. 2, pp. 427-451, 2002.

[17] M. Chapman, "Frequency-dependent anisotropy due to mesoscale fractures in the presence of equant porosity," Geophysical Prospecting, vol. 51, no. 5, pp. 369-379, 2003.

[18] X. Wu, M. Chapman, X. Y. Li, and P. Boston, "Quantitative gas saturation estimation by frequency-dependent amplitudeversus-offset analysis," Geophysical Prospecting, vol. 62, no. 6, pp. 1224-1237, 2014.

[19] J. G. Berryman and H. F. Wang, "Elastic wave propagation and attenuation in a double-porosity dual- permeability medium," International Journal of Rock Mechanics and Mining Sciences, vol. 37, no. 1-2, pp. 63-78, 2000.

[20] V. Grechka, P. Mazumdar, and S. A. Shapiro, "Predicting permeability and gas production of hydraulically fractured tight sands from microseismic data," Geophysics, vol. 75, no. 1, pp. B1-B10, 2010.

[21] C. Ecker, J. Dvorkin, and A. Nur, "Sediments with gas hydrates: internal structure from seismic AVO," Geophysics, vol. 63, no. 5, pp. 1659-1669, 1998.

[22] A. Buland and H. Omre, "Bayesian linearized AVO inversion," Geophysics, vol. 68, no. 1, pp. 185-198, 2003.

[23] X. Pan, G. Zhang, and X. Yin, "Elastic impedance variation with angle and azimuth inversion for brittleness and fracture parameters in anisotropic elastic media," Surveys in Geophysics, vol. 39, no. 5, pp. 965-992, 2018.
[24] L. Zhou, J. Li, X. Chen, X. Liu, and L. Chen, "Prestack amplitude versus angle inversion for Young's modulus and Poisson's ratio based on the exact Zoeppritz equations," Geophysical Prospecting, vol. 65, no. 6, pp. 1462-1476, 2017.

[25] W. Alemie and M. D. Sacchi, "High-resolution three-term AVO inversion by means of a Trivariate Cauchy probability distribution," Geophysics, vol. 76, no. 3, pp. R43-R55, 2011.

[26] R. Gehrmann, C. Müller, P. Schikowsky, T. Henke, M. Schnabel, and C. Bönnemann, "Model-based identification of the base of the gas hydrate stability zone in multichannel reflection seismic data, Offshore Costa Rica," International Journal of Geophysics, vol. 2009, Article ID 812713, 12 pages, 2009.

[27] K. Aki and P. G. Richards, Quantitative Seismology: Theory and Methods, University Science Books, San Francisco, 1980.

[28] R. T. Shuey, "A simplification of the Zoeppritz equations," Geophysics, vol. 50, no. 4, pp. 609-614, 1985.

[29] G. C. Smith and P. M. Gidlow, "Weighted stacking for rock property estimation and detection of gas," Geophysical Prospecting, vol. 35, no. 9, pp. 993-1014, 1987.

[30] J. L. Fatti, G. C. Smith, P. J. Vail, P. J. Strauss, and P. R. Levitt, "Detection of gas in sandstone reservoirs using AVO analysis: a 3-D seismic case history using the Geostack technique," Geophysics, vol. 59, no. 9, pp. 1362-1376, 1994.

[31] L. Zhi, S. Chen, and X. Li, "Amplitude variation with angle inversion using the exact Zoeppritz equations-theory and methodology," Geophysics, vol. 81, no. 2, pp. N1-N15, 2016.

[32] Z. Zong, X. Yin, and G. Wu, "Geofluid discrimination incorporating poroelasticity and seismic reflection inversion," Surveys in Geophysics, vol. 36, no. 5, article 9330, pp. 659-681, 2015.

[33] D. Grana, T. Fjeldstad, and H. Omre, "Bayesian Gaussian mixture linear inversion for geophysical inverse problems," Mathematical Geosciences, vol. 49, no. 4, pp. 493-515, 2017.

[34] B. Goodway, T. Chen, and J. Downton, "Improved AVO fluid detection and lithology discrimination using Lamé petrophysical parameters; " $\lambda \rho$ ", " $\mu \rho$ ", \& " $\lambda / \mu$ fluid stack", from P and S inversions," in SEG Technical Program Expanded Abstracts 1997, pp. 183-186, Society of Exploration Geophysicists, 1997.

[35] B. H. Russell, D. Gray, and D. P. Hampson, "Linearized AVO and poroelasticity," Geophysics, vol. 76, no. 3, pp. C19-C29, 2011.

[36] B. H. Russell, K. Hedlin, F. J. Hilterman, and L. R. Lines, "Fluid-property discrimination with AVO: a Biot-Gassmann perspective," Geophysics, vol. 68, no. 1, pp. 29-39, 2003.

[37] A. Alshangiti, "Frequency-dependent AVO inversion: comparison of different AVO approximations," in SEG Technical Program Expanded Abstracts 2019, pp. 724-728, Society of Exploration Geophysicists, 2019.

[38] A. Wilson, M. Chapman, and X. Y. Li, "Frequency-dependent AVO inversion," in SEG Technical Program Expanded Abstracts 2009, pp. 341-345, Society of Exploration Geophysicists, 2009.

[39] X. Wu, M. Chapman, A. Wilson, and X.-Y. Li, "Estimating seismic dispersion from pre-stack data using frequencydependent AVO inversion," in SEG Technical Program Expanded Abstracts 2010, pp. 425-429, Society of Exploration Geophysicists, 2010.

[40] C. Luo, G. Huang, and X. Li, "Frequency-dependent AVO inversion based on sparse constrained inversion spectral decomposition," in SEG Technical Program Expanded 
Abstracts 2017, pp. 524-528, Society of Exploration Geophysicists, 2017.

[41] J. Liu, J. Ning, X. Liu, C. Y. Liu, and T. S. Chen, “An improved scheme of frequency-dependent AVO inversion method and its application for tight gas reservoirs," Geofluids, vol. 2019, Article ID 3525818, 12 pages, 2019.

[42] Z. Zhang, X. Y. Yin, and Z. Y. Zong, “A new frequencydependent AVO attribute and its application in fluid identification," in 75th EAGE Conference \& Exhibition incorporating SPE EUROPEC 2013, European Association of Geoscientists \& Engineers, June 2013.

[43] R. Chen, X. Chen, J. Li, Z. Wang, and B. Wang, "A new hydrocarbon indicator derived from FAVO inversion," in SEG Technical Program Expanded Abstracts 2016, pp. 516-520, Society of Exploration Geophysicists, 2016.

[44] K. Wang, X. Chen, D. Wang et al., "A new insight into the hydrocarbon indicator of tight sandstone reservoir," in SEG Technical Program Expanded Abstracts 2018, pp. 401-405, Society of Exploration Geophysicists, 2018.

[45] Y. Wang, X. Ma, H. Zhou, and Y. Chen, "L1- 2 minimization for exact and stable seismic attenuation compensation," Geophysical Journal International, vol. 213, no. 3, pp. 1629-1646, 2018.

[46] M. Chapman, E. Liu, and X. Y. Li, "The influence of fluidsensitive dispersion and attenuation on AVO analysis," Geophysical Journal International, vol. 167, no. 1, pp. 89-105, 2006.

[47] E. Fjar, R. M. Holt, A. M. Raaen, R. Risnes, and P. Horsrud, Petroleum Related Rock Mechanics, Elsevier, 2008.

[48] D. C. Bonar and M. D. Sacchi, "Complex spectral decomposition via inversion strategies," in SEG Technical Program Expanded Abstracts 2010, pp. 1408-1412, Society of Exploration Geophysicists, 2010.

[49] S. Yuan, Z. Yu, H. Gao et al., "Semi-blind time-variant sparse deconvolution using a weighted L1 norm," in 81st EAGE Conference and Exhibition 2019, European Association of Geoscientists \& Engineers, June 2019.

[50] E. Van Den Berg and M. P. Friedlander, "Probing the Pareto frontier for basis pursuit solutions," SIAM Journal on Scientific Computing, vol. 31, no. 2, pp. 890-912, 2009.

[51] D. Gray, B. Goodway, and T. Chen, "Bridging the gap: using AVO to detect changes in fundamental elastic constants," in SEG Technical Program Expanded Abstracts 1999, pp. 852855, Society of Exploration Geophysicists, 1999.

[52] G. Partyka, J. Gridley, and J. Lopez, "Interpretational applications of spectral decomposition in reservoir characterization," The Leading Edge, vol. 18, no. 3, pp. 353-360, 1999.

[53] E. Odebeatu, J. Zhang, M. Chapman, E. Liu, and X. Y. Li, "Application of spectral decomposition to detection of dispersion anomalies associated with gas saturation," The Leading Edge, vol. 25, no. 2, pp. 206-210, 2006.

[54] H. Liu, J. Li, X. Chen, B. Hou, and L. Chen, "Amplitude variation with offset inversion using the reflectivity method," Geophysics, vol. 81, no. 4, pp. R185-R195, 2016.

[55] Z. Zong, X. Yin, and G. Wu, "AVO inversion and poroelasticity with P-and S-wave moduli," Geophysics, vol. 77, no. 6, pp. N17-N24, 2012. 

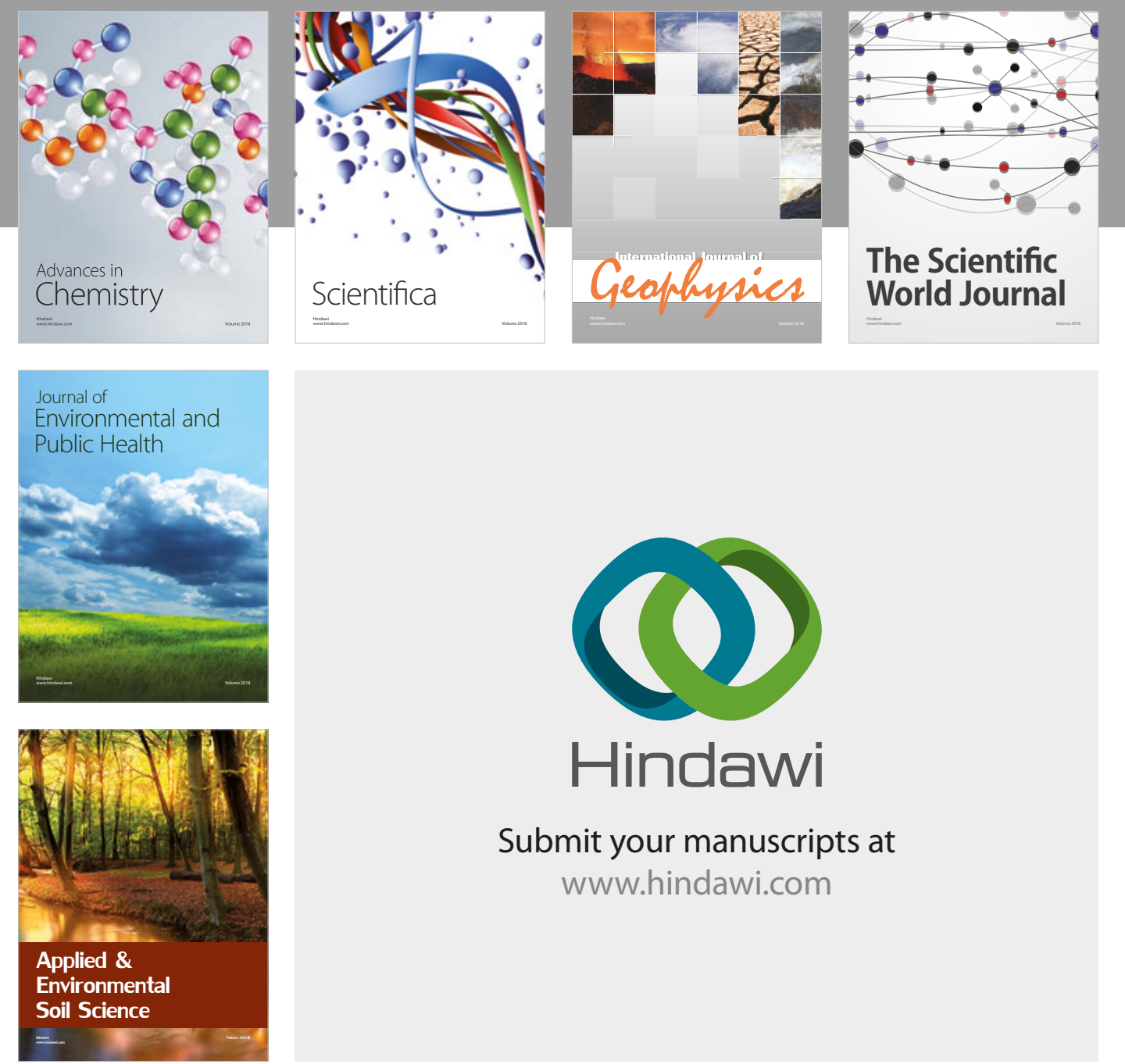

The Scientific

\section{World Journal}
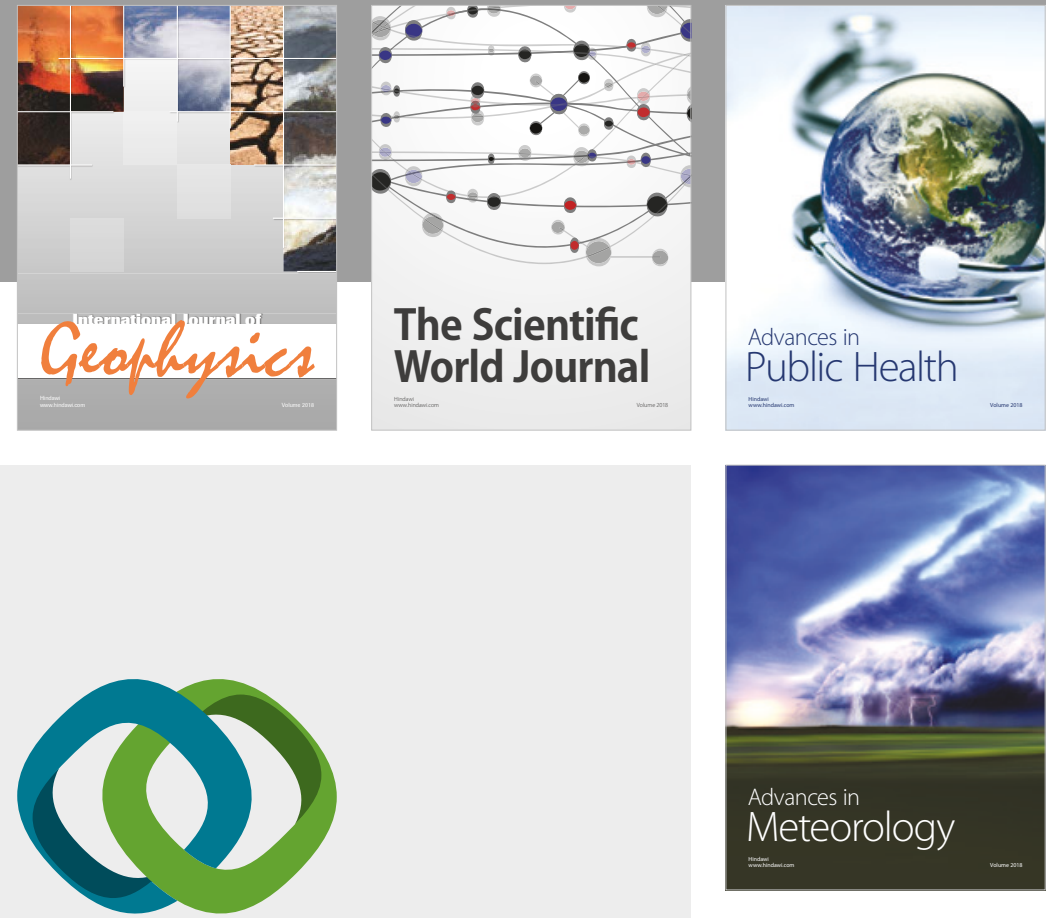

Advan

Public Health

\section{Hindawi}

Submit your manuscripts at

www.hindawi.com
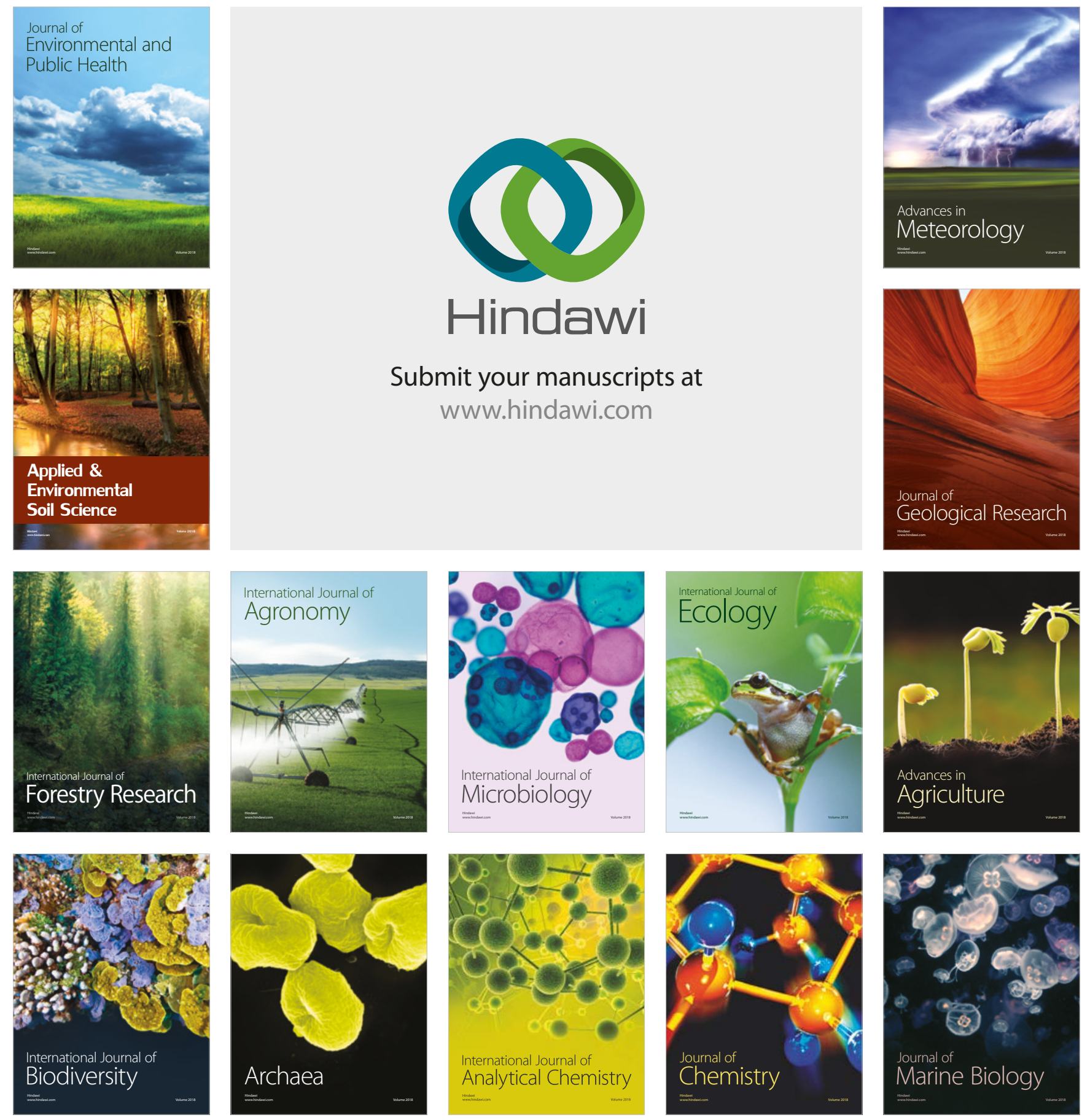